\title{
Developing organisational systems and culture to support evidence-based practice: the experience of the Evidence-Based Ward Project
}

\begin{abstract}
The advancement of research based practice has been a goal in nursing for many years. Evidence-based practice (EBP) provides a framework and process for the systematic incorporation of research evidence and patient preference into clinical decision making at the level of the individual practitioner and the healthcare organisation. Few well designed studies have tested the effectiveness of organisational strategies for encouraging the systematic use of research in nursing practice, ${ }^{1}$ and more research is needed into the reality and consequences of adopting EBP. ${ }^{2}$

In 1996, an acute National Health Service (NHS) hospital trust and a university department in London, UK began a joint project to increase the systematic use of research by nurses in clinical practice. EBP was selected as the approach, and the hospital-wide project began in late 1996. The project was facilitated by a senior lecturer appointed jointly by the 2 organisations, with half of his time allocated for this role. A diagnostic assessment done early in the project identified the need for the development of organisational and individual capacity to support and use EBP. ${ }^{3}$ The Evidence-Based Ward Project used action research to explore ways in which the organisation and culture of practice in a busy acute ward could be developed to make EBP part of the "normal" approach to practice. The project ran for 10 months and was coordinated by the new ward manager and the senior lecturer.
\end{abstract}

\section{Project site}

When the project began, the hospital was a fairly typical UK NHS district general hospital, with approximately 520 beds. During the project, the hospital merged with a neighbouring NHS trust of similar size. The hospital had a history of providing nurse training but had only recently become a clinical placement site for medical students. The ward was selected as typical of other wards in the hospital, with 25 beds and an average length of stay of 4.5 days. The staffing establishment included 15 registered nurses and 5 unqualified nurses. The ward staff participated voluntarily.

\section{Project design}

The design was based on research evidence that suggests that tailored, multifaceted interventions targeting different barriers to change are most consistently effective for facilitating organisational and practitioner changes. ${ }^{45}$ Introducing systems to support EBP is a major change process that requires restructuring of the local clinical environment and a change in the prevailing culture of practice. ${ }^{67}$ The project used action research ${ }^{8}$ and work redesign. ${ }^{9}$ Ward staff selected and prioritised the areas they wished to tackle. To ensure that the project was realistic, minimal additional resources were made available.

The change team, whose role was to identify problems and to find solutions, comprised a nominated member from each grade of nursing staff, the pharmacist, occupational therapist, and discharge liaison nurse linked to the ward team. The task of developing the cultural and organisational systems to support EBP was included in the team's remit. The facilitators supported the team by encouraging them to use the work redesign model and action planning as a change process.

The concept of the "link nurse" already existed in the ward and in the hospital as a whole. The role had traditionally been conceptualised as one of practice development, but no clear operational definition existed. As part of the project, the link nurse role was defined as one of addressing the EBP questions generated by the ward team. The 5 stage EBP process to be used was as follows:

- Formulate an EBP question

- Systematically search for evidence

- Critically appraise the evidence

- Change practice where indicated

- Evaluate the effects of change.

Pairs of nurses took responsibility for answering a specific question, and if a change of practice was indicated took the lead in the change process. The senior lecturer provided 1.5 days of additional training for this role; in small groups on the ward, EBP questions were generated on the basis of existing patients. Nurses were given prepared tools to help with the process of finding and critically appraising evidence. Additional resources for the photocopying and storage of research articles were provided.

Clinical supervision (colleagues help each other to reflect on and learn from practice) was not operational in the setting when the project began, and there were no trained clinical supervisors in the hospital trust. The new ward manager had clinical supervision experience and initially took on the role of clinical supervisor for all ward staff. The nursing staff were given a reflective practice diary that included the headings used in Johns' model of structured reflection. ${ }^{10}$ Subsequently, more individual sessions were held, and some of the more senior staff started to provide some clinical supervision to members of their team. A trust-wide training programme provided additional external supervisors for some members of the ward team as the project progressed.

\section{Evaluating the project}

The project was complex, involving different layers of intervention with many participants. It was exploratory in the sense that the task was to develop the organisational systems and culture to support EBP without knowing what these would be in this particular context. In these circumstances, it was difficult to know which outcomes to measure. As such, the evaluation was driven by a need to reconcile relevance and rigour within the context of a sociotherapeutic relationship, in which the aim was to facilitate desired change. ${ }^{11} 12$

Indicators of the culture and quality of care on the ward and of application of the EBP process were selected; previously 
collected data were also used. Process indicators included staff sickness, client complaints, and the number of EBP questions and answers generated. Proxy indicators included participating nurses' perceptions of the impact of the project and the quality of patient documentation assessed using a modified version of MONITOR 2000 before and after the intervention. ${ }^{13}$

\section{Results}

Several developments occurred in the organisation and culture of practice during the project. They included:

- The introduction of team and named nursing (each patient has their own named lead nurse)

- The introduction of patient assessment and care planning using the activities of daily living model as a key activity for all qualified staff, which resulted in improved patient documentation

- The introduction of a standardised format for patient handover, including the identification of EBP questions

- Improvements in the ward environment (eg, floors were relaid and piped oxygen was connected)

- The introduction of team based and self rostering

- The introduction and enforcement of personnel management policies (eg, individual performance review).

The developments selected and methods of organisation implemented were strongly influenced by the ward manager's priorities and previous experience.

During the course of the project, the ward staff identified 22 EBP questions. One nurse completed a critically appraised topic summary with practice recommendations for her EBP question on the care of percutaneous endoscopic gastrostomy sites. This was a substantial piece of work that involved fairly comprehensive searches of Medline, CINAHL, and the Cochrane Library; contacting subject experts; and critically appraising and summarising the evidence. Much of this was done outside of work time.

The nurses who participated in the project reported an increase in confidence in their own and their colleagues' abilities. They said that they had developed and enhanced their existing communication, organisation and management, leadership, and teamwork skills. The documentation of patient care as assessed by MONITOR scores also improved (mean difference in pre-test and post-test scores 7.6\%, 95\% CI 2.8 to 12.4). The average sickness rate among nurses was lower during the project than during an equivalent period in the previous year (mean difference $10 \mathrm{~d} / \mathrm{mo}, 95 \%$ CI 5.3 to 14.3).

The organisational changes that occurred during the project were sporadic, and many were not sustained. The systems that were set up to encourage the nurses to systematically question practice and find evidence-based answers to their questions (primarily the link nurse scheme) did not become fully operational. With a few exceptions, enthusiasm for and active participation in the project remained confined to change team members. To some degree, all nurses were resistant to change, but the topics about which they were resistant varied. A small group of nurses (primarily those who worked night duty, part time, or who had worked on the ward for many years) actively resisted almost all the changes agreed to by their colleagues. This group appeared to gain little if anything from the project, despite the efforts of the facilitators to get them involved. Developments on the ward since the completion of the project suggest that changes that did occur were fragile and easily reversed.
Lessons learnt from the project

The changes did not reach the stage that Lewin described as refreezing. ${ }^{14}$ Why was change so difficult and why were the changes made not sustained? Several important factors that restricted putting EBP into operation seemed to originate in current structural (ie, institutional and professional) contexts.

RESOURCES AND CONTROL OF WORKLOAD

During periods when the ward was understaffed, all the nurses complained of being "too busy," and project activity during these periods was minimal. For example, if too few of the ward's own nurses were working on a particular shift, new systems of practice could not work. Change team members were often too busy to attend team meetings. Training sessions for link nurses were difficult to organise because staff could not be freed up to attend them. These external factors, however, did not affect everyone in the same way. There was variation within and among the nurses in their response to the perceived workload.

\section{SELECTION AMONG COMPETING PRIORITIES}

During the project, EBP had to compete with other priorities within the trust, such as reducing waiting lists and improving responses to patient complaints. Regional managers did not monitor specific aspects of EBP in the trust with the same degree of scrutiny as the meeting of waiting list targets. This may have affected the capacity of the institution to support and promote change in the participating ward, and how staff selected their priorities. Examples of "bad practice," when highlighted to ward staff by someone they perceived to be an authority figure, often produced an immediate response from the ward staff. For example, feedback from the director of nursing to all wards about the number of reported drug administration errors highlighted 2 drug administration errors (no patient harm involved) in 1 month on the participating ward. In response, the ward manager changed the system for giving drugs on the ward. This change consumed much time and energy, and the new system increased the use of nursing resources. By contrast, nurses did not have a similar urgent response to EBP questions that emanated from reflection on practice, even when they could not justify a particular practice.

\section{SKILLS AND KNOWLEDGE FOR EBP}

Unexpectedly, the nurses came to the project with low baseline knowledge of EBP. All participants had great difficulty identifying and framing a clinical question. All had few searching and critical appraisal skills. The training and ongoing support was inadequate to address these deficits. The participants made varying progress in the extent to which they grasped the principles of EBP. Nurses who qualified more recently appeared to make the most progress. The reasons are unclear, but it seems likely to be partly a result of their willingness and ability to work on their questions outside of work time. Available time during working hours was often accounted for by competing priorities.

\section{WARD MANAGER AS A ROLE MODEL}

The ward manager appeared to be a key figure in the changes that were achieved. Her clinical and management abilities were instrumentally important, but she also served as a role model. Although she was a positive role model in many areas of practice, she seemed to be a negative role model for EBP. She did not complete her EBP question and gave her role as a link nurse a lower priority than other aspects of her role. This may have had a negative role effect on the perceptions of the other nurses about the importance of EBP. 
Future work on the implementation of EBP

The results of the project correspond with findings from other studies of change in the organisation of nursing practice, which suggest that structural, organisational factors will be key determinants of the successful implementation of changes. ${ }^{15}{ }^{16}$ This is likely to be the case whether the change involves a small, specific aspect of practice or a large cultural shift as here. The project showed that a substantial shift in organisational and cultural thinking is necessary to get nurses to make research evidence part of routine practice. The findings also suggest that individual clinical units will find it difficult to make EBP operational in the absence of a change in current professional and organisational contexts. Local factors that appear important include the existence of a functional clinical team with clearly defined roles and responsibilities. Issues that are primarily structural rather than local in origin include:

- The priority that organisations place on EBP (eg, the effective scrutiny and feedback of EBP in individual trusts by clinicians and those in positions of authority)

- The matching of resources to workloads (eg, the inclusion of clinical practice development when considering the workload of individual units and clinicians)

- The adequate preparation of clinical leaders and nurses with the skills and knowledge for EBP, including time to practise their use

- The ongoing development of qualified nurses' generic, problem solving, information technology, and critical thinking skills.

MARK NEWMAN, RGN, MA, PGCHE Research Centre for Transcultural Studies in Healthcare Middlesex University and Barnet E Chase Farm Hospitals NHS Trust, UK IRENA PAPADOPOULOS, RN, RM, MA(Ed), $\mathrm{PhD}$ Research Centre for Transcultural Studies in Healthcare Middlesex University, UK
RITA MELIFONWU, RGN, MSC Barnet \& Chase Farm Hospitals NHS Trust, UK

This study was funded by the participating institutions and the Foundation of Nursing Studies. The authors would like to thank the staff of Durban ward and other participants from the 2 hospitals and UNN Commercial Enterprises Ltd for permission to use "Monitor 2000." The views expressed in this article are solely the responsibility of the authors.

1 Le May A, Mulhall A, Alexander C. Bridging the research-practice gap: exploring research cultures of practitioners and managers. $J$ Adv Nurs 1998;28:428-37.

2 Mulhall A. Nursing, research and the evidence [editorial]. Evidence-Based Nursing 1998;1:4-6.

3 Newman M, Papadopoulos I, Sigsworth J. Barriers to evidence based practice. Clinical Effectiveness in Nursing 1998;2:11-20.

4 Grol R, Grimshaw J. Evidence-based implementation of evidence-basedmedicine. Joint Commission Journal on Quality Improvement 1999;25:503-13.

5 NHS Centre for Reviews and Dissemination. Getting evidence into practice. Effective Healthcare 1999;5:1-16.

6 Stocking B. Promoting change in clinical care. Quality in Health Care 1992;1:56-60.

7 Lomas J. Retailing research: increasing the role of evidence in clinical services for childbirth. Millbank Quarterly 1993;71:439-75.

8 Hart E, Bond M. Action research for health and social care. Buckingham: Open University Press, 1995.

9 Schumacher C. The Barry neighbourhood hospital: concepts and scopes. Godstone: Work Structuring Ltd, 1992.

10 Johns C. Framing learning through reflection within Carper's fundamental ways of knowing in nursing. J Adv Nurs 1995;22:226-34.

11 Gregory D, Martin S. Crafting evaluation research in the public sector: reconciling rigour and relevance. British Journal of Management 1994;5(special issue): $\mathrm{S} 43-52$

12 Menzies IEP. The functioning of social systems as a defence against anxiety. London: Tavistock Institute, 1970.

13 Fearon MM, Goldstone LA. MONITOR 2000. Newcastle: Unique Business Services, 1992.

14 Lewin K. Field theory in social sciences. New York: Harper \& Row, 1951.

15 Procter $\mathrm{S}$. The functioning of nursing routines in the management of a transient workforce. J Adv Nurs 1989;14:180-9.

16 Adams A, Bond S, Hale CA. Nursing organizational practice and its relationship with other features of ward organization and job satisfaction. J Adv Nurs 1998;27:1212-22.

\section{Acknowledgments}

As our third year of publication draws to a close, we would like to thank the many people who have contributed their time, expertise, and enthusiasm to Evidence-Based Nursing.

To our panel of advisers on qualitative research, who helped to select qualitative research papers, edited abstracts and commentaries for selected files*, and who continue to help us to develop our approach to abstracting qualitative research:

*Caroline Carlisle, Liverpool, UK

*Marlene Z Cohen, Houston, Texas, USA

Marilyn Ford-Gilboe, London, Ontario, Canada

*Jenny Ploeg, Hamilton, Ontario, Canada

*RitaSchreiber, Victoria, British Columbia, Canada

*Carl Thompson, York, UK

*Sally Thorne, British Columbia, Canada

Judith Wuest, Fredericton, New Brunswick, Canada

To the individuals who helped us in the selection of quantitative studies that were clinically important and relevant to practising nurses in their areas of expertise, and those who edited abstracts and commentaries for selected files:

*Heather Arthur, Hamilton, Ontario, Canada

Cathy Buffett, Hamilton, Ontario, Canada
Wendy Goodine, Etobicoke, Ontario, Canada Theresa Harper, Hamilton, Ontario, Canada Jeannette LeGris, Hamilton, Ontario, Canada *Ann Mohide, Hamilton, Ontario, Canada Brenda Morgan, London, Ontario, Canada * Janet Pinelli, Hamilton, Ontario, Canada

To Brian Hutchison, who provided thoughtful, methodological advice about selected studies, and to Geoff Norman, who assisted with the formulation of definitions included in the glossary and provided statistical advice on selected studies.

To the people who served as peer reviewers for the EBN Notebook and Implementation Forum, for their thoughtful and timely feedback:

Jennifer Blythe, Hamilton, Ontario, Canada

Kate Flemming, York, UK

Carolyn Ingram, Hamilton, Ontario, Canada

Dorothy McCaughan, York, UK

Andrea Nelson, York, UK

Geoff Norman, Hamilton, Ontario, Canada

Carl Thompson, York, UK

And finally, to the many people who generously contributed their time and expertise in the preparation of commentaries. 